Coulomb integrals $[i i \mid j j]$ was necessary in order to obtain good agreement with experiment. ${ }^{2}$ In this Note, it is shown that, within the formalism suggested by Ruedenberg, a different determination of $\zeta$ and $\zeta^{C}$ is possible, which leads to acceptable agreement between the predicted and observed properties of benzene, without any ad hoc adjustment of theoretically computed integrals:

(1) The potential $U_{i}^{C}\left(\zeta^{C}\right)$ has been replaced by $W_{i}\left(\zeta, \zeta^{C}\right)$ :

$$
W_{i}=U_{i}^{C}+e^{2}\left[\left(\phi_{i}(\zeta)\right)^{2} \mid-e^{2}\left[\left(\phi_{i}\left(\zeta^{C}\right)\right)^{2} \mid,\right.\right.
$$

because it is inconsistent to use two different exponents in describing the same $2 p_{z}$ distribution.

(2) The separations between the lower excited electronic states of benzene have been calculated ${ }^{3}$ in the manner described by Ruedenberg. The values given in Table I are purely theoretical, and are determined by the value of $\zeta$. Evidently, values of $\zeta$ in the region of 1.2 lead to reasonable agreement with experiment.

(3) When a value of $\zeta$ is assumed and the integrals $[i i \mid j j]$ and overlap integrals determined, the value of the resonance integral $\gamma$ can be deduced ${ }^{3}$ from each of the spectroscopic transition energies. With $\zeta=1.2$, one obtains the following values (in electron volts) for $\gamma$ :

$$
\begin{array}{llll}
-1.45 & \left({ }^{1} A_{1 g} \rightarrow{ }^{1} E_{1 u}\right) ; & -2.05 & \left({ }^{1} A_{1 g} \rightarrow{ }^{1} B_{2 u}\right) ; \\
-2.12 & \left({ }^{1} A_{1 g} \rightarrow{ }^{3} B_{1 u}\right) ; & -2.17 & \left({ }^{1} A_{1 g} \rightarrow{ }^{3} E_{1 u}\right) ; \\
-2.31 & \left({ }^{1} A_{1 g} \rightarrow{ }^{1} B_{1 u}\right) & &
\end{array}
$$

The mean value is $\bar{\gamma}=-2.02 \mathrm{eV}$. This semiempirical result is not very sensitive to the value of $\zeta$ assumed for its derivation. Thus, with $\zeta=1.6, \bar{\gamma}=-2.31$, the individual values of $\gamma$ being scattered in the range $-2.93 \leq \gamma \leq-1.30 \mathrm{eV}$.

(4) Theoretical values of $\gamma$ have been calculated, ${ }^{3}$ using the assumed potential $W\left(\zeta, \zeta^{C}\right)$ : calculations have been carried out for the values of $\zeta$ and $\zeta^{C}$ in the range 1.0 to 1.6 , at intervals of 0.05 . Some results are this approach, however, empirical adjustment of shown in Table II. This table also shows the theoretical

TABLE I. Separation (in electron volts) between excited electronic levels of benzene.

\begin{tabular}{lccccc}
\hline \hline Separation & $\zeta=1.0$ & $\zeta=1.2$ & $\zeta=1.4$ & $\zeta=1.6$ & Expt1 \\
\hline${ }^{1} E_{1 u^{-3} B_{1 u}}$ & 4.16 & 4.96 & 5.81 & 6.70 & 3.2 \\
${ }^{1} B_{1 u^{-3} B_{1 u}}$ & 1.16 & 1.91 & 2.80 & 3.81 & 2.4 \\
${ }^{1} B_{2 u}-{ }^{3} B_{1 u}$ & 0.81 & 1.29 & 1.85 & 2.44 & 1.1 \\
${ }^{3} E_{1 u}-{ }^{3} B_{1 u}$ & 0.41 & 0.65 & 0.93 & 1.22 & 0.7 \\
\hline
\end{tabular}

a Theory refers to $(0,0)$ transitions, which are difficult to locate experimentally. Experimental data are taken from M. J. S. Dewar and N. Sabelli, J. Phys. Chem. 66, 2310 (1962) with the following exceptions: (a) $\Delta E\left({ }^{1} A_{1} \rightarrow\right.$ ${ }^{8} B_{1 u}$ is taken as $3.8 \mathrm{eV}$ instead of the more usual $3.6 \mathrm{eV}$, to allow for distortion in the triplet state [J. De Heer and R. Pauncz, J. Chem. Phys. 39, 2314

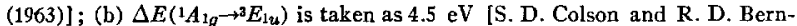
stein, J. Chem. Phys. 43, 2661 (1965)].
TABLE II. Results of calculations (all energies in electron volts).

$\begin{array}{lrrrrr}\zeta & 1.2 & 1.2 & 1.3 & 1.3 & 1.6 \\ \zeta^{\mathrm{c}} & 1.4 & 1.5 & 1.4 & 1.5 \ldots & 1.1 \\ & & & & & \\ \gamma^{\mathrm{a}} & -1.67 & -1.85 & -2.01 & -2.19 & -2.22 \\ I_{A} & 9.62 & 8.30 & 9.67 & 8.19 & 16.72 \\ & -1.15 & -2.93 & -1.89 & -3.97 & +4.67\end{array}$

${ }^{a} \gamma$ is the purely theoretical value of the resonance integral. $I$ and $A$ are the theoretical values of the ionization potential and electron affinity of benzene, which may be compared with the experimental value [R. E. Honig, J. Chem. Phys. 16, $105(1948)] I=9.43 \mathrm{eV}$ and the estimated [N. S. Hush and J. A. Pople, Trans. Faraday Soc. 51, $600(1955)] A \approx-0.54 \mathrm{eV}$.

values of the electron affinity and ionization potential calculated $^{3}$ in the manner described by Ruedenberg. ${ }^{1,2}$ Best agreement between theory and experiment is obtained with $\zeta \approx 1.2, \zeta \approx 1.4$, the agreement being as good as could be expected with the present orbital approximation. On the other hand, with the Ruedenberg values of $\zeta$ and $\zeta^{C}$ quoted above, (a) the calculated spacings of the excited electronic levels are in all cases about $100 \%$ too large; (b) the calculated ionization potential is about $7 \mathrm{eV}$ too large; (c) the electron affinity is predicted to have the very unlikely value of $4.7 \mathrm{eV}$.

(5) The integral $\left\langle\phi_{i}\left|\mathcal{H}_{i}{ }^{C}\right| \phi_{i}\right\rangle$ is sensitive to the choice of values of $\zeta$ and $\zeta^{C}$; with $\zeta=1.2$ and $\zeta^{C}=1.4$, its value is $4.30 \mathrm{eV}$. It has been customary to equate $\left\langle\phi_{i}\left|\mathcal{H}_{i}{ }^{C}\right| \phi_{i}\right\rangle$ to the negative of the $s p^{3}$ valence state $p$-electron affinity of carbon $(\sim 0.5 \mathrm{eV})$, but the present results indicate that this cannot be done. This is due partly to the noninclusion of exchange operators in $\mathfrak{K}_{i}{ }^{C}$, and partly to the fact that an electronic structure derived with $2 s, 2 p_{x}, 2 p_{y}$ (but not $2 p_{z}$ ) orbitals as functions of the same exponent $\xi^{C}$ must be a poor approximation to the "valence state" in the sense that Mulliken" used the term.

${ }^{1}$ K. Ruedenberg, J. Chem. Phys. 34, 1861, 1878, 1884, 1892, 1907 (1961)

${ }^{2}$ (a) K. Ruedenberg and E. M. Layton, Jr., J. Chem. Phys. 34, 1897 (1961). (b) R. L. Hummel and K. Ruedenberg, J. Phys. Chem. 66, 2334 (1962).

${ }^{3}$ To avoid possible error for small values of $\zeta$, integrals dependent on overlap between nonneighboring atoms have been included throughout the calculations reported in this note. See Ref. 1, pp. 1878-1883.

${ }^{4}$ R. S. Mulliken, J. Chem. Phys. 2, 782 (1934).

\section{Dipole Moment of Difluorophosphine}

\author{
J. G. Morse and R. W. Parry \\ Department of Chemistry, The University of Michigan \\ Ann Arbor, Michigan \\ (Received 12 December 1966)
}

$\mathbf{T}$ IHE recently reported ${ }^{1}$ compound, difluorophosphine, $\mathrm{F}_{2} \mathrm{HP}$, is a much stronger electron-pair donor than either $\mathrm{PH}_{3}$ or $\mathrm{PF}_{3}$. As a result, the borane addition compound of $\mathrm{F}_{2} \mathrm{HP}, \mathrm{F}_{2} \mathrm{HPBH}_{3}$, is significantly less dissociated at $25^{\circ} \mathrm{C}$ than are the related compounds, 
TABLE I. Data for determination of dipole moment of gaseous $\mathrm{F}_{2} \mathrm{HP}$."

\begin{tabular}{|c|c|c|}
\hline 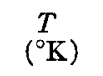 & $\begin{array}{c}\left(\Delta C / \Delta P_{P=0}\right) \\
{\left[(\mu \mu \mathrm{F} / \mathrm{mm}) \times 10^{3}\right]}\end{array}$ & $\begin{array}{l}P_{T} \\
(\mathrm{cc})\end{array}$ \\
\hline 299.01 & 1.649 & 48.06 \\
\hline 287.56 & 1.774 & 49.70 \\
\hline 277.24 & 1.876 & 50.69 \\
\hline 267.90 & 2.002 & 52.26 \\
\hline 258.77 & 2.133 & 53.79 \\
\hline 250.61 & 2.268 & 55.38 \\
\hline
\end{tabular}

B Polarization(atomiotelectronio) $=10.7 \pm 1.3 \mathrm{cc} ; C_{s}=213.3 \pm 0.5 ;$ slope of line $P_{T}$ vs $(1 / T)=11170 \pm 340$.

$\mathrm{H}_{3} \mathrm{BPH}_{3}$ and $\mathrm{H}_{3} \mathrm{BPF}_{3}{ }^{2}$ A recently completed investigation of some of the physical characteristics of $\mathrm{F}_{2} \mathrm{HP}$ shows further that the molecule has a significantly larger dipole moment $(\mu=1.35 \pm 0.02 \mathrm{D})$ than either $\mathrm{F}_{3} \mathrm{P}(\mu=1.025 \mathrm{D})^{3}$ or $\mathrm{H}_{3} \mathrm{P}(\mu=0.579) .{ }^{4}$

Gaseous $\mathrm{F}_{2} \mathrm{HP}$ was prepared by methods reported earlier. ${ }^{1}$ The dielectric constant of $\mathrm{HPF}_{2}$ gas was measured as a function of temperature using a conventional heterodyne beat system, ${ }^{5}$ operating at 1 $\mathrm{Mc} / \mathrm{sec}$ over the temperature range $250.61^{\circ}-299.01^{\circ} \mathrm{K}$. The capacitance cell used was like that described by Holmes and Carter. ${ }^{6}$ Its capacitance was determined using $\mathrm{NH}_{3}$ gas as a standard, the value of $1.468 \mathrm{D}$ being taken as the dipole moment and $5.3 \mathrm{cc}$ as the temperature-independent part of the polarization. ${ }^{7}$ Pertinent data are summarized in Table I. The dipole moment was obtained by the standard Debye method.

Values for the $\mathrm{P}-\mathrm{H}$ and $\mathrm{P}-\mathrm{F}$ bond moments can be calculated from the geometry and known dipole values for $\mathrm{PH}_{3}$ and $\mathrm{PF}_{3}$ if one assumes that the lone pair moment in each case is negligible. ${ }^{8}$ If the measured $\mathrm{F}-\mathrm{P}-\mathrm{F}$ angle of $98.2 \pm 0.6^{\circ}$ in $\mathrm{PF}_{3}{ }^{9}$ is used, the $\mathrm{P}-\mathrm{F}$ bond moment can be calculated as $0.71 \mathrm{D}$. Similar calculations for $\mathrm{PH}_{3}$ using the known HPH bond angle of $93.3^{\circ}{ }^{10}$ give a $\mathrm{P}-\mathrm{H}$ bond moment of $0.36 \mathrm{D}$. Addition of these vectors at the $\mathrm{PF}_{3}$ angle gives a resultant of $1.09 \mathrm{D}$ for the molecule $\mathrm{F}_{2} \mathrm{HP}$. Addition of the vectors at the $\mathrm{PH}_{3}$ angle gives a resultant of $1.08 \mathrm{D}$. Clearly, small changes in molecular geometry are not adequate to account for the discrepancy between the calculated moment and the observed value of $1.35 \pm 0.02 \mathrm{D}$.

Agreement between measured and calculated values can be obtained if it is assumed that slight changes in the $\mathrm{P}-\mathrm{F}$ bond moment occur when fluorines in $\mathrm{PF}_{3}$ are replaced by hydrogens in the sequence $\mathrm{PF}_{3-x} \mathrm{H}_{x}$. If a reasonable FPF angle of $96^{\circ}$ is assumed in $\mathrm{F}_{2} \mathrm{HP}$ and the value of the $\mathrm{P}-\mathrm{H}$ bond moment is assumed to be constant in the series at a value of $0.36 \mathrm{D}$, the value of $\mu_{\mathrm{P}-\mathrm{F}}$ calculated for $\mathrm{HPF}_{2}$ is $0.94 \mathrm{D}$. In an earlier report on the dipole moments of the alkyl phosphines, ${ }^{8}$ it was necessary to assume stepwise change in the $P-R$ moment as one proceeds from $\mathrm{PR}_{3}$ to $\mathrm{PRH}_{2}$. This same procedure is required in the present study. Values for the two cases are summarized in Table II. It is appropriate to note, however, that in the calculations of the alkyl phosphines, the phosphorus was assumed to be the negative end of the dipole vector, while in the fluorophosphines the fluorine was assumed to be negative with respect to phosphorus and hydrogen positive relative to phosphorus.

The reproducibility of the trends is striking. The compound $\mathrm{H}_{2} \mathrm{PF}$ has not yet been synthesized but the extrapolated P-F moment from Table II can be used

TABLE II. Empirical values of $\mathrm{P}-\mathrm{R}$ bond moments which by vector addition give measured moments of alkyl phosphines and fluorophosphines.

\begin{tabular}{|c|c|c|c|}
\hline Compound & $-\mathrm{CH}_{3}$ & $\begin{array}{c}\mathrm{R}= \\
-\mathrm{C}_{2} \mathrm{H}_{5}\end{array}$ & $-F$ \\
\hline $\mathrm{PH}_{2} \mathrm{R}$ & $1.06 \mathrm{D}$ & $1.17 \mathrm{D}$ & $1.15^{\mathrm{a}}$ \\
\hline $\mathrm{PHR}_{2}$ & 0.95 & 1.06 & 0.94 \\
\hline $\mathrm{PR}_{\mathbf{3}}$ & 0.83 & 0.94 & 0.71 \\
\hline
\end{tabular}

a Estimated.

to estimate the over-all moment of the still unknown molecule $\mathrm{H}_{2} \mathrm{FP}$ as $1.30 \mathrm{D}$.

A qualitative electronic argument to rationalize the empirically observed trend in $\mathrm{P}-\mathrm{F}$ moments can be given. In $\mathrm{PF}_{3}$ each highly electronegative fluorine atom competes with two other fluorines for negative charge from the phosphorus. Replacement of a fluorine by a less electronegative hydrogen permits greater migration of charge from phosphorus toward the two competing fluorines thus increasing the net P-F bond moment in $\mathrm{HPF}_{2}$.

This work was supported in part by the U. S. Public Health Service Research Grant No. CA-07989-02 from the National Cancer Institute.

${ }^{1}$ R. W. Rudolph and R. W. Parry, Inorg. Chem. 4, 1339 (1965).

2 R. W. Rudolph and R. W. Parry, "Fluorophosphine Ligands IV Base Strength Difluorophosphine," J. Am. Chem. Soc. (to be published).

${ }^{3}$ R. G. Shulman, B. P. Dailey, and C. H. Townes, Phys. Rev. 78, 145 (1950).

4 M. H. Sirvetz and R. E. Weston, J. Chem. Phys. 21, 898 (1953).

${ }^{5}$ J. G. Morse, Ph.D. dissertation, University of Michigan, Ann Arbor, Mich. (1966).

${ }_{6}^{6}$ R. R. Holmes and R. P. Carter, Jr., J. Chem. Phys. 43, 1645 (1965).

7 A. A. Maryott and F. Buckley, Natl. Bur. Std. (U.S.), Circ. No. 537 (1953).

${ }^{8}$ G. Kodama, J. R. Weaver, J. LaRochelle, and R. W. Parry, Inorg. Chem. 5, 710 (1966).

${ }^{9} \mathrm{O}$. L. Hersh, Ph.D. dissertation, University of Michigan (1963).

${ }_{10}$ D. K. Coles, W. E. Good, J. K. Bragg, and A. H. Sharbough, Phys. Rev. 82, 877 (1951). 